Original Article

\title{
PHOTOLYTIC DEGRADATION STUDY ON FILM COATED ATENOLOL TABLET AVAILABLE IN MARKET
}

\author{
SABBIR AHMED*, MAHABUBUR RAHAMAN, MOHAMMED FAISAL BIN KARIM, MD. ANISUR RAHMAN
}

Department of Pharmacy, East-West University, A/2, Jahurul Islam Avenue, Aftabnagar, Dhaka-1212, Bangladesh

Email: sabbirahmed52@ymail.com

Received: 06 Jul 2018 Revised and Accepted: 14 Aug 2018

\begin{abstract}
Objective: Despite its sensitivity to light, atenolol has been manufactured as a film-coated tablet with normal blister packaging by several pharmaceutical companies in Bangladesh. The aim of this study was to investigate the extent of light-induced potency degradation of a randomly
\end{abstract} selected film coated brand of atenolol.

Methods: Atenolol tablets were exposed to different lighting conditions such as normal room light, direct sunlight and different incandescent lights $(25 \mathrm{~W}$ and $40 \mathrm{~W})$. Subsequently, UV spectroscopy technique was employed to determine the relative reduction of light absorbance compared to their respective controls. Thereafter, photolytic degradation was calculated by means of the potency reduction of tablets.

Results: In all lighting conditions, atenolol tablets underwent exposure dependent gradual decrease in potency. Except for normal room light condition, a significant decrease in potency was found even after 4 to $6 \mathrm{~h}$ of exposure to all lighting conditions. After $6 \mathrm{~h}$, potency reduction was found at $40-47 \%, 26-38 \%$ and $34-36 \%$ in the samples exposed to direct sunlight, $25 \mathrm{~W}$ bulb, and $40 \mathrm{~W}$ bulb respectively. Although the shelf life of the film coated tables was $2 \mathrm{y}$, surprisingly, statistically significant reduction in potency was observed within only $30 \mathrm{~d}$ in room light condition.

Conclusion: In order to protect from light, blister packaging is not sufficient for film coated atenolol tablets. Photo-stability of all brands of atenolol must be ensured either by protective packaging materials or by optimizing the formulations.

Keywords: Hypertension, Atenolol, Photolytic degradation, Therapeutic inactivity

(C) 2018 The Authors. Published by Innovare Academic Sciences Pvt Ltd. This is an open-access article under the CC BY license (http://creativecommons.org/licenses/by/4.0/) DOI: http://dx.doi.org/10.22159/ijpps.2018v10i10.26794

\section{INTRODUCTION}

A common medical condition, hypertension (HTN), or high blood pressure (HBP), is affecting more than $75 \%$ of older people( $>70 \mathrm{y})$, whereas one-third of a young adult has been estimated suffering from HBP [1]. According to a new study [2], it was the biggest risk factor that led to the global disease burden in 2010. Moreover, hypertensive heart disease and HTN contribute about 17.3/100000 of the yearly age-adjusted deaths as mentioned in the US government's Centers for Disease Control and Prevention (CDC) [3] lists. Most common comorbidities of HTN patients include higher levels of serum cholesterol, low-density lipoprotein (LDL), triglycerides and body mass index (BMI) [4]. In medical literature, HTN often coined as 'silent killer' since it does not cause any severe symptoms to appear [5]. However, a cross-sectional study performed in Jammu has concluded that most of the hypertensive patients (72.5\%) were concerned about the symptoms [6], alternatively, another study conducted in Kerala has suggested that the majority of hypertensive patients lack the knowledge on stroke prevention [7]. Although cardiac walking and laughter therapy has recently been found as maintaining optimum blood pressure in HTN patients $[8,9]$, one of the major successes in medicine in the past decades was the treatment of HTN. The striking therapeutic advancement provided a greater capability of controlling blood pressure in hypertensive patients [10]. Interestingly, recent studies have revealed that advanced drug formulation techniques such as transdermal system, floating bead formulations, and bi-layer tablet formulations can be used for more prolonged and effective treatment of HTN [11-13].

Since 1960, $\beta$-blockers has been using as the most promising treatment of HTN compared to existing antihypertensive drugs available, including guanethidine, ganglionic blocker or methyldopa [3]. A selective $\beta 1$ receptor antagonist, atenolol, was developed in 1976 as a better alternative for non-selective $\beta$-blocker propranolol in the treatment of HTN. In addition, being unable to cross the bloodbrain barrier, it has drawn more attraction exerting less central nervous system side effects [14]. Atenolol, considering as the most widely used $\beta$ adrenergic receptor blocking agent, has often been the drug of choice for reference in randomized controlled trials of HTN [15].

Currently, drug photo-stability investigation has drawn much attention since photolytic degradation can reduce the potency of a drug and also form toxic degradation products contributing in adverse effects. As a consequence, light-sensitive drugs and adjuvants are recommended to be protected from light during storage [16] accomplishing by using an opaque blister, opaque gelatin shell or opaque film packaging for tablets or capsules [17]. Besides, a reflective pigment or a pharmaceutical dye with the same absorption spectrum as the drug principle can be added in the formulation to obtain the opaqueness [18]. Photophysical and photochemical properties of a drug are indispensable to predict phototoxic properties and also to evaluate product quality. Photo-stability and phototoxicity of some classes of drugs have been studied [19]. Although atenolol has been reported as photosensitive [16], in Bangladesh, many brands of this drug have been manufacturing with transparent blister packaging or as film coated. In our current study, we investigated the extent of lightinduced potency degradation of a randomly selected film coated brand of atenolol after exposing to different lighting conditions.

\section{MATERIALS AND METHODS}

Photo-stability study of drug formulations is carried out assuming that the finished product will experience various lighting conditions such as normal room light, direct sunlight and artificial electric bulb light. Noteworthy, reasonable sensitivity, simplicity and cost-effectiveness have lead spectrophotometric techniques to be the most preferred method for routine analysis [20]. As a consequence, we treated our test brand of atenolol by different light sources to determine potency reduction employing UV spectrophotometry.

\section{Sample collection}

The study was conducted using a randomly selected brand of atenolol (batch number: 14004) tablet formulation, purchased from 
a local pharmacy in Dhaka, Bangladesh. All tablets were manufactured in blister packs, containing $50 \mathrm{mg}$ Atenolol each. In order to ensure reproducibility of the results, tablets were divided into three different groups (A, B, and C). In each group of tablets, some were kept as a control in a light-protected amber container, and the rest were treated at different exposure conditions.

\section{Preparation of standard curve}

$0.1 \mathrm{~N}$ sulfuric acid $\left(\mathrm{H}_{2} \mathrm{SO}_{4}\right)$ solution was prepared from $98 \% \mathrm{w} / \mathrm{v}$ stock solution of sulfuric acid (Analar, United Kingdom) to determine the wavelength of maximum absorption, $\lambda_{\max }(223.5 \mathrm{~nm})$ using a reference standard of Atenolol (99.5\% potency) supplied by ACI pharmaceuticals, Bangladesh. Nine different concentrations from 0.001 to $0.009 \mathrm{mg} / \mathrm{ml}$ of atenolol were prepared by using $0.1 \mathrm{~N}$ $\mathrm{H}_{2} \mathrm{SO}_{4}$. Subsequently, the absorbances were measured for each concentration at $\lambda_{\max }(223.5 \mathrm{~nm})$, and the graph was plotted against their respective concentrations to yield the standard curve (as shown in fig. 1) with the following equation:/Where, $y=$ absorbance, $\mathrm{x}=$ concentration of the $\operatorname{drug}(\mathrm{mg} / \mathrm{ml})$. Potencies of atenolol formulations after different treatment conditions were calculated using this equation (fig. 1) after measuring absorbance by UV spectroscopy.

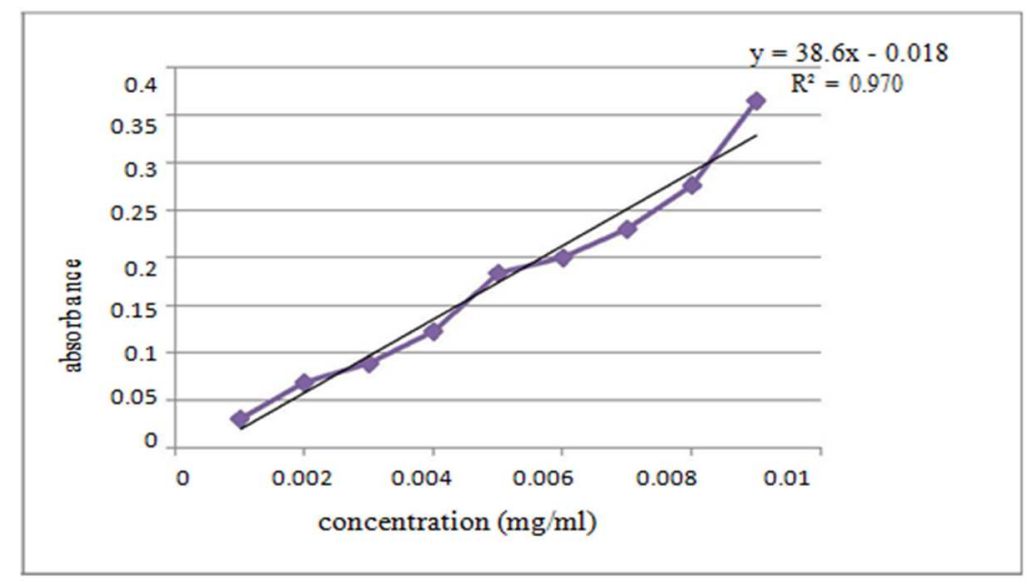

Fig. 1: Plot showing a straight line for absorbance with respect to concentration for atenolol. The $R^{2}$ represents coefficient of determination

\section{Normal room light exposure}

The experiment was designed to observe any change in potency of atenolol tablet formulation following exposure to normal room light condition for $60 \mathrm{~d}$. From each group (A, B and C) of tablets, 10 were kept in a light protected container as control and 40 tablets were kept under a normal room light condition for $60 \mathrm{~d}$. Those were analyzed by removing 10 tablets each time in 15, 30, 45 and $60 \mathrm{~d}$ to determine their level of potency by UV spectroscopy (Shimadzu UV 1800, Japan).

\section{Direct sunlight exposure}

Experiments were performed to study how direct sunlight affects the potency of light unprotected atenolol tablet formulations. From each group (A, B and C), 10 tablets were kept in a light protected container as control and 30 tablets were exposed to direct sunlight for $7.5 \mathrm{~h}$ at a stretch. The minimum and maximum average temperatures of the experiment days were recorded as 30 and $32{ }^{\circ} \mathrm{C}$. Every two hours intervals 10 tablets were removed and temperatures were recorded by a thermometer (Midwest Home brewing and Winemaking Supplies, USA) up to $6 \mathrm{~h}$ (as shown in table 1) to determine their potency level by UV spectroscopy.

\section{Exposure to incandescent light bulbs (25 $\mathrm{W}$ and $40 \mathrm{~W}$ )}

The same procedure was followed to determine the changes in the potency of atenolol tablet formulations after exposure to $25 \mathrm{~W}$ incandescent light bulbs (Philips, Bangladesh) and $40 \mathrm{~W}$ incandescent light bulbs (Philips, Bangladesh). Temperatures were also recorded similarly (table 2).

Table 1: Withdrawal temperatures at different time intervals after exposure to direct sunlight

\begin{tabular}{|c|c|c|c|}
\hline No. of tablets from each group & Collected sample & Withdrawal intervals (h) & Temperature $\left({ }^{\circ} \mathrm{C}\right)$ \\
\hline \multirow[t]{3}{*}{$40(10$ for control) } & 10 & 2 & 30 \\
\hline & 10 & 4 & 31 \\
\hline & $10+10$ (control) & 6 & 32 \\
\hline
\end{tabular}

Table 2: Withdrawal temperatures at different time intervals after exposure to $25 \mathrm{~W}$ and $40 \mathrm{~W}$ bulbs

\begin{tabular}{lll}
\hline Withdrawal intervals (h) & Temperature ( $\left.{ }^{\circ} \mathbf{C}\right)$ & $\mathbf{2 5 ~ W}$ \\
\hline 2 & 27 & 30 \\
4 & 27 & 30 \\
6 & 30 & 32 \\
\hline
\end{tabular}

\section{Assay procedure}

After termination of treatment, tablets from both controls and samples underwent the same analytical procedure. Those were crushed into powder and weighed in order to measure the average weight of the single respective tablet. Subsequently, that weighed powder was made into a solution by diluting 1000 times into $0.1 \mathrm{~N}$ sulfuric acid. Absorbances of the solutions were then measured by UV spectroscopy and the respective potencies of each sample and control were calculated by standard curve calculation (fig. 1). 


\section{Statistical analysis}

Statistical significance was studied with Students t-test. For all statistical analyses, a two-tailed p-value was measured, and it was presented for individual experiments considering significant when the value was less than $0.05\left({ }^{*} \mathrm{p}<0.05,{ }^{* *} \mathrm{p}<0.01\right.$ and $\left.{ }^{* * *} \mathrm{p}<0.001\right)$.

\section{RESULTS AND DISCUSSION}

Loss of potency is the most luminous result of drug photodecomposition, which can turn a drug formulation therapeutically inactive. However, minor degradation of a drug product during storage and administration can lead to adverse effects, which is very common apparently [21]. Since the forced degradation of drug products differ in the mechanism of degradation reaction from normal condition [22], we followed both strategies to investigate the photosensitivity of atenolol more extensively.

A common trend of gradual decrease in potency with higher duration of treatment has shown in all groups of samples in all treatment conditions (as shown in fig. 2, 3, 4 and 5). However, the extent of potency reduction varies in between groups and treatment conditions.

Exposure to normal room light condition for $60 \mathrm{~d}$ resulted highly statistically significant $\left({ }^{* * *} \mathrm{p}<0.001\right)$ decrease in potency (up to $23 \%$ approximately) of atenolol compared to control in both groups (fig. 2 ). Although the samples exposed for $15 \mathrm{~d}$ to normal room light condition did not experience any significant change, those exposed for 30 and $45 \mathrm{~d}$ experienced a statistically significant reduction $\left({ }^{* *} \mathrm{p}<0.01,{ }^{* * *} \mathrm{p}<0.001\right)$ in potency (fig. 2$)$. Minor variations of the extent of statistical significance for samples exposed for 30 and $45 \mathrm{~d}$ among different groups are because of different standard deviations. It could also be a result of variations in the purity of active pharmaceutical ingredient (API) among different tablets. Since the intensity of the radiation acts as a rate-limiting factor of photolytic degradation [23], undersized but not unusual, those minor differences in potency reduction among different groups could also be due to a nonhomogeneous mixing of API with excipients.

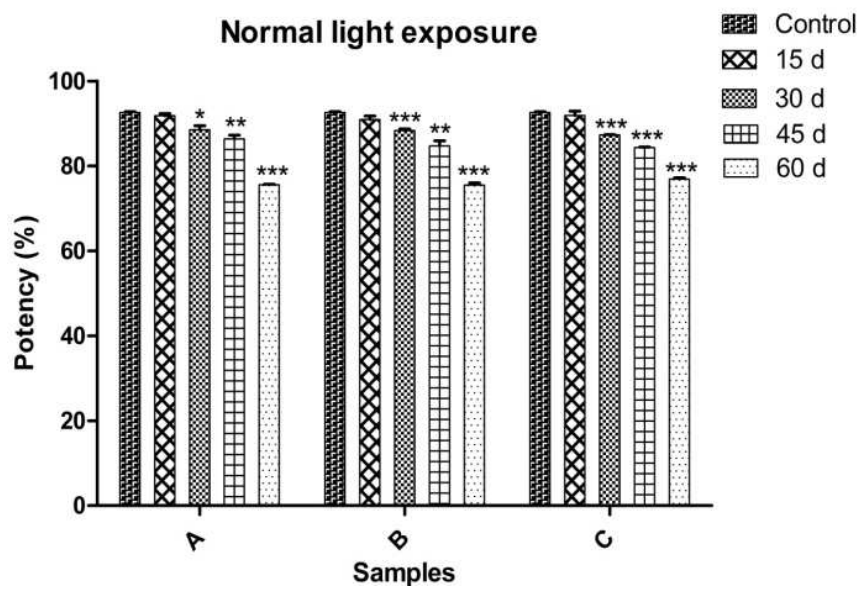

Fig. 2: Potency of three different groups (A, B, and C) of a randomly selected brand of atenolol tablet formulation after exposure to normal room light for 15, 30, 45 and $60 \mathrm{~d}$. Results are presented as percent compared to control (unexposed sample), and each result is represented as mean \pm SD of triplicate experiments. Error bars indicate standard deviations. Asterisk indicates statistically significant change. ${ }^{*} \mathbf{p}<0.05,{ }^{* *} \mathbf{p}<0.01, * * * p<0.001$

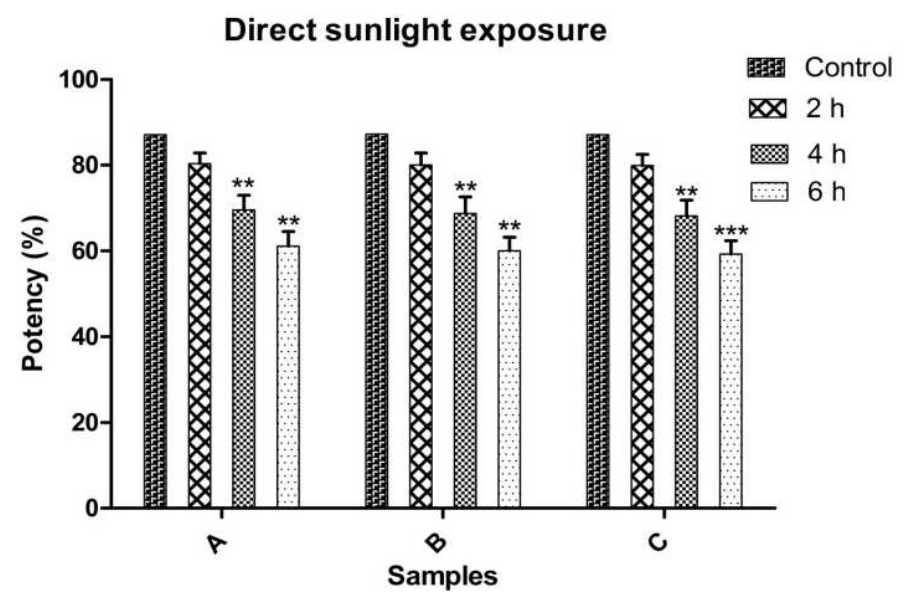

Fig. 3: Potency of three different groups (A, B, and C) of a randomly selected brand of atenolol tablet formulation after exposure to direct sunlight for 2, 4 and $6 \mathrm{~h}$. Results are presented as percent compared to control (unexposed sample), and each result is represented as mean $\pm S D$ of triplicate experiments. Error bars indicate standard deviations. Asterisk indicates statistically significant change. ${ }^{*} p<0.05$, ${ }^{* *} \mathbf{p}<0.01,{ }^{* * *} \mathbf{p}<0.001$

Forced photolytic degradation was carried out by exposing the samples and controls to direct sunlight and electric bulb lights. As shown in the fig. 3 , all the three groups of the sample have shown a similar trend of gradual decrease in potency (40-47\%) with exposure time. Additionally, samples exposed for 4 and $6 \mathrm{~h}$ to direct sunlight have found statistically significantly decreased in potency $\left({ }^{* *} \mathrm{p}<0.01, * * * \mathrm{p}<0.001\right)$ compared to respective controls in both groups (fig. 3). 


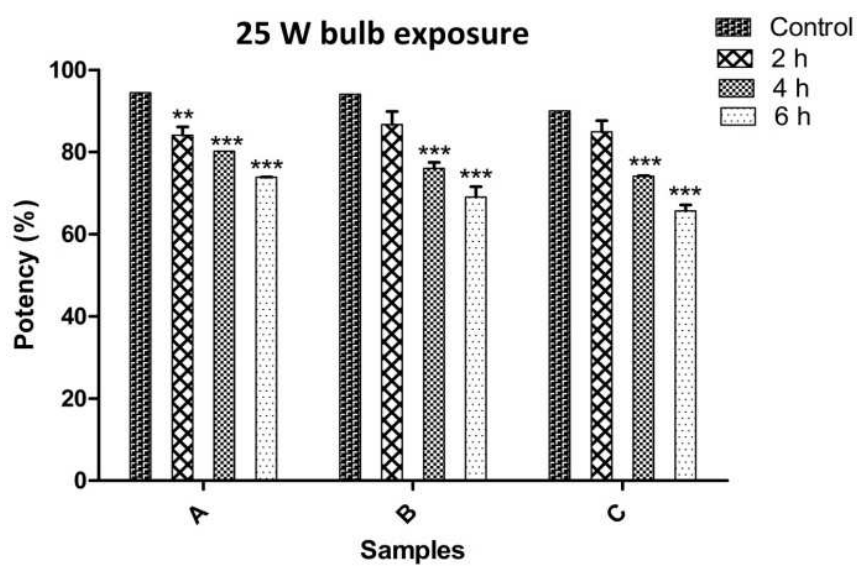

Fig. 4: Potency of three different groups (A, B, and C) of a randomly selected brand of atenolol tablet formulation after exposure to $25 \mathrm{~W}$ incandescent light bulbs for 2,4 and $6 \mathrm{~h}$. Results are presented as percent compared to control (unexposed sample) and each result is represented as mean $\pm S D$ of triplicate experiments. Error bars indicate standard deviations. Asterisk indicates statistically significant change. ${ }^{*} \mathbf{p}<0.05, * * \mathbf{p}<0.01, * * * \mathbf{p}<0.001$

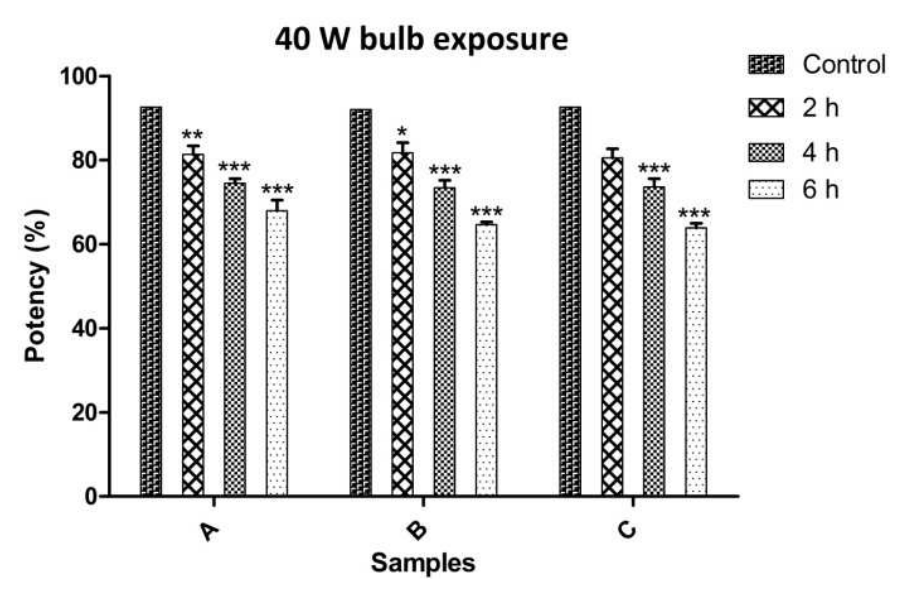

Fig. 5: Potency of three different groups (A, B, and C) of a randomly selected brand of atenolol tablet formulation after exposure to $40 \mathrm{~W}$ incandescent light bulbs for 2,4 and $6 \mathrm{~h}$. Results are presented as percent compared to control (unexposed sample), and each result is represented as mean \pm SD of triplicate experiments. Error bars indicate standard deviations. Asterisk indicates statistically significant change. ${ }^{*} \mathbf{p}<0.05, * * \mathbf{p}<0.01, * * * \mathbf{p}<0.001$

Although exposure to $25 \mathrm{~W}$ and $40 \mathrm{~W}$ incandescent lights resulted in a similar motif of gradual degradation of the potency of atenolol with time in both cases and in all sample groups, notably, exposure to 40 $\mathrm{W}$ bulbs experienced more significant degradation of potency (34$36 \%$ ) for 6 h exposure. However, in both cases, samples exposed to 4 and 6 h have shown a highly significant reduction $(* * * p<0.001)$ in potency compared to their respective controls. Combination of all results displays a very clear exposure dependent pattern of photolytic degradation of a randomly selected film coated brand of atenolol after exposure to any lighting condition (normal room light, direct sunlight and incandescent bulb light), warning that film coating of atenolol with normal blister packaging is not enough to protect it from photolytic degradation.

Apart from therapeutic inactivity by reducing potency, photolytic degradation of atenolol can also contribute to alteration of physicochemical properties of the formulation [21]. Moreover, photolytic degradation of atenolol produces a minor toxic product, 2-(4hydroxyphenyl) acetamide, which may cause gastrointestinal irritation, nausea, vomiting, and diarrhea [16]. Therefore atenolol should be manufactured with adequate light protection in order to achieve the desired therapeutic effect and to avoid adverse effects as well.

Notwithstanding that it is possible to implement by replacing the packaging materials with light protective ones, changes in formulation could also be another path to address this problem. The Photolytic degradation of a tablet mostly takes place on the upper surface that contacts with incident light [21]. Alternatively, irrespective of exposure time, the interior of the tablet remains almost unaffected since light radiation barely reaches there. Therefore, the factors influencing depth of light penetration, for example, the thickness of powder bed, particle size, color, and thickness could also be optimized to avoid photolytic degradation of a tablet [17].

\section{CONCLUSION}

Several different brands of atenolol are available in the pharma market of Bangladesh, which have not been manufactured with proper light protection. Critical analysis of the results of our current study and knowledge from literature allow us to conclude that atenolol must be manufactured with sufficient light protection to retain therapeutic activity throughout its shelf life. Formulation difficulties for light-sensitive drugs are not uncommon. However, a good understanding of photolytic degradation mechanism can contribute to accomplishing optimum stability of a formulation.

\section{ACKNOWLEDGMENT}

This study was supported by the Department of Pharmacy, East West University, Bangladesh.

\section{AUTHORS CONTRIBUTIONS}

The first author has done an analysis and wrote the manuscript. The second author has executed all experiments, and the last two authors have designed the study. 


\section{CONFLICT OF INTERESTS}

There are no conflicts of interest

\section{REFERENCES}

1. [WHO] World Health Organization. A Global Brief on Hypertension: Silent Killer, Global Public Health Crisis. Geneva: WHO Press; 2013.

2. Lim SS, Vos T, Flaxman AD, Danaei G, Shibuya K, Adair-Rohani $\mathrm{H}$, et al. A comparative risk assessment of burden of disease and injury attributable to 67 risk factors and risk factor clusters in 21 regions, 1990-2010:a systematic analysis for the Global Burden of Disease Study 2010. Lancet 2012;380:222460.

3. Andros V, Egger A, Dua U. Blood pressure goal attainment according to JNC 7 guidelines and utilization of antihypertensive drug therapy in MCO patients with type 1 or type 2 diabetes. J Manag Care Pharm 2006;12:303-9.

4. Dwivedi S, Gonmei Z, Toteja G, Srivastava N. Assessment of risk factors of hypertension among adults residing in an urban slum of Delhi. Asian J Pharm Clin Res 2018;11:405-7.

5. Moore J. Hypertension: catching the silent killer. J Pract Nurs 2005;30:16-35.

6. Sharma S, Sawhney V. Awareness, stress, anxiety, and depression among hypertensive patients attending cardiac outpatient department in a super specialty hospital. Asian J Pharm Clin Res 2016;9:62-4.

7. Missiriya S, John J. Assess the prevalence of hypertension and knowledge regarding the prevalence of stroke. Asian J Pharm Clin Res 2017;10:177-80.

8. Santhanasamy PMC. The effectiveness of cardiac walking on blood pressure among patients with hypertension at a selected hospital in Kancheepuram district. Asian J Pharm Clin Res 2017;10:171-4.

9. Santhanasamy PJJ. Effectiveness of laughter therapy on blood pressure among patients with hypertension. Asian J Pharm Clin Res 2017;10:246-50.

10. Makridakis S, DiNicolantonio JJ. Hypertension: empirical evidence and implications in 2014. Open Heart 2014; 1:e000048.
11. Shukla R, Omray LK. Formulation characterization of diltiazem transdermal system for the treatment of hypertension. Int J Curr Pharm Res 2016;8:12-5.

12. Agarwal S, Zamil F, Singh L, Saxena A. Formulation and evaluation of floating beads of diltiazem HCL. Int J Curr Pharm Res 2016;8:38-42.

13. Das M, Sahu B, Hazarika J. Development of bilayer tablets for the immediate and controlled release of allicin. Int J Curr Pharm Res 2017;9:153-60.

14. Agon P, Goethals P, Van Haver D, Kaufman JM. Permeability of the blood-brain barrier for atenolol studied by positron emission tomography. J Pharm Pharmacol 1991;43:597-600.

15. Medical Research Council trial of treatment of hypertension in older adults: principal results. MRC Working Party. Br Med J 1992;304:405-12.

16. Andrisano V, Gotti R, Leoni A, Cavrini V. Photodegradation studies on atenolol by liquid chromatography. J Pharm Biomed Anal 1999;21:851-7.

17. Thoma K, Klimek R. Photostabilization of drugs in dosage forms without protection from packaging materials. Int J Pharm 1991;67:169-75.

18. Matsuda Y, Itooka T, Mitsuhashi Y. Photo-stability of indomethacin in model gelatin capsules: effects of film thickness and concentration of titanium dioxide on the coloration and photolytic degradation. Chem Pharm Bull (Tokyo) 1980;28:2665-71.

19. Albini A, Fasani E. editors. Drugs, photochemistry and photostability. Cambridge: Royal Society of Chemistry; 1998.

20. El-Didamony A, Hafeez S, Saad A. Extractionspectrophotometric determination of some antihypertensive drugs in a pharmaceutical and biological fluid using two sulphonphthalein dyes. Int J Appl Pharm 2015;7:10-7.

21. Tonnesen HH. Formulation and stability testing of photolabile drugs. Int J Pharm 2001;225:1-14.

22. Bakshi M, Singh S. Development of validated stability-indicating assay methods-- a critical review. J Pharm Biomed Anal 2002;28:1011-40

23. Hotha KK, Reddy SPK, Raju VK, Ravindranath LK. Forced degradation studies: practical approach-overview of regulatory guidance and literature for the drug products and drug substances. Int Res J Pharm 2013;4:78-85. 